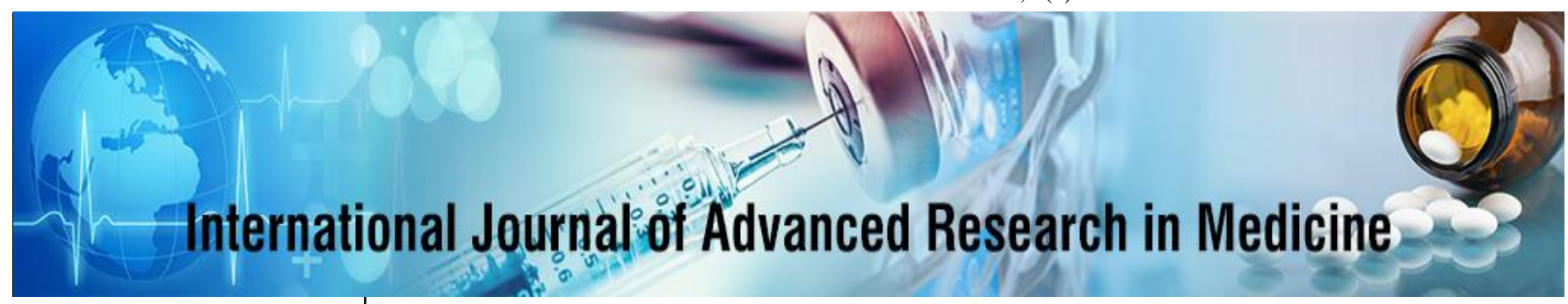

E-ISSN: 2706-9575 P-ISSN: 2706-9567 IJARM 2020; 2(2): 271-274 Received: 30-05-2020 Accepted: 05-07-2020

Dr. Shailendra Singh Rajpoot MD Medicine,

Metro Hospital and Cancer Research Center Jabalpur, Madhya Pradesh, India
Corresponding Author: Dr. Shailendra Singh Rajpoot MD Medicine,

Metro Hospital and Cancer Research Center Jabalpur, Madhya Pradesh, India

\section{Clinico-hematological profile in patients diagnosed with dengue fever: A prospective study}

\author{
Dr. Shailendra Singh Rajpoot
}

DOI: $\underline{\text { https://doi.org/10.22271/27069567.2020.v2.i2d.130 }}$

\begin{abstract}
Aim: The aim of the study was to determine the clinical and haematological profile in patients with Dengue fever.

Materials and Methods: A prospective study was conducted in the Department of General Medicine, Metro Hospital and Cancer Research Centre, Jabalpur, MP, India from Jan 2019 to Jan 2020. Total 120 patients with complaints of fever and clinical features of dengue with positive NS1 antigen test or dengue antibody serology IgM or IgG or both were included in the study.

Results: Most of the cases (47.5\%) were seen in the 20-30 year age group followed by 40-50 years $20.83 \%, 30-40$ years $15 \%$. Majority of the patients were males $78(65 \%)$ compared to females, 42 $(35 \%)$ and the male to female ratio was 1.8:1. Fever was the most common presentation and was seen in 58 cases $(48.33 \%)$ cases . $54(45 \%)$ cases showed $\mathrm{Hb}$ of $9-12 \mathrm{gm} \%$, followed by $38(31.67 \%)$ cases showed $\mathrm{Hb}$ of $12-15 \mathrm{gm} \%, 9(7.5 \%)$ had $\mathrm{Hb}$ of below $9 \mathrm{gm} \%$ and $19(15.83 \%)$ had $\mathrm{Hb}$ of above 15 gm\% .63(52.5\%) cases showed hematocrit of $25-35 \%$ and $35(29.17 \%)$ showed hematocrit of $35-$ $45 \% .21(17.5 \%)$ cases, count of $4000-11000$ cells/cu $\mathrm{mm}$ seen in $81(67.5 \%)$ cases and $>11000 /$ cumm was seen in 18 cases (15\%) as per .out of 120 cases of dengue fever, $87.5 \%$ cases had thrombocytopenia and $12.5 \%$ cases had severe thrombocytopenia $(<20,000 / \mathrm{cumm})$ with bleeding manifestation.

Conclusion: Hemoconcentration, leukopenia, thrombocytopenia, and raised liver enzymes SGOT and SGPT along with reactive/ plasmacytoid lymphocytes on peripheral smear gives enough clues to test for dengue serology so that dengue cases can be diagnosed in their initial stages.
\end{abstract}

Keywords: Dengue fever, hemoconcentration, liver enzymes

\section{Introduction}

Dengue infection is one of the most common mosquito borne viral diseases of public health significance. It is caused by one of the four serotypes of the dengue virus (DEN-1, DEN-2, DEN-3 and DEN-4) also referred to as an arbovirus (arthropod-borne viruses) that belongs to the genus Flavivirus of the family Flaviviridae ${ }^{[1-2]}$. The virus serotypes are closely related but antigenically distinct. It is a disease with a wide clinical spectrum and a wide variety of presentations, ranging from asymptomatic to an undifferentiated fever to the more severe life threatening forms such as Dengue hemorrhagic fever (DHF)/ dengue shock syndrome (DSS). ${ }^{[3]}$ Transmission to humans occurs by the bite of the female Aedes aegypti mosquito infected by one of four serotypes of the virus. This mosquito, a domestic species adapted to urban conditions, is the main vector in India. In recent decades, the incidence of dengue infection has increased around the world and has become a major international public health concern [4]. An estimated 50 million dengue infections occur worldwide annually and approximately 2.5 billion people, i.e., two-fifth of world's population in tropical and subtropical countries are at risk. The reported case fatality rate in India is $3-5 \%{ }^{[5-6]}$. Most developing countries have epidemics of febrile illnesses including typhoid, measles, leptospirosis, and severe acute respiratory distress syndrome that can be confused with dengue due to similar clinical features ${ }^{[7]}$. Of biochemical variables, the most frequent changes occur in liver function tests such as in serum aspartate aminotransferase (AST), serum alanine aminotransferase (ALT), Gamma-glutamyl transpeptidase and alkaline phosphatase levels, and serum albumin concentrations ${ }^{[8-10]}$. The period of transmission from humans to mosquitoes begins one day before the start of fever up to the sixth day of illness corresponding to the viremia phase. After a female Aedes mosquito bites an individual in the viremia phase, viral replication (extrinsic incubation) begins in the vector from eighth to twelve days. 
In humans, the incubation period ranges from 3 to 15 days (intrinsic incubation) with an average of 5 days ${ }^{[11]}$.

\section{Materials and Methods}

A prospective study was conducted in the Department of General Medicine, India from Jan 2019 to Jan 2020, after taking the approval of the protocol review committee and institutional ethics committee. After taking informed consent detailed history was taken from the patient or the relatives.

\section{Methodology}

Total 120 patients with complaints of fever and clinical features of dengue with positive NS1 antigen test or dengue antibody serology IgM or IgG or both were included in the study. Age, gender, clinical presentation, duration of fever, dehydration, hemodynamic status, urine output, hepatomegaly, ascites, pleural effusion, presence of petechiae, positive tourniquet test, other bleeding manifestations, hematocrit and platelet count were recorded at presentation. Febrile patients with positive NS1 antigen or IgM or both on rapid card tests. IgG may be positive or negative were include in this study. Patients with only IgG positive on rapid card tests were excluded from the study. Patients with other identified illnesses like typhoid, malaria which were coexisted with dengue positive serology were excluded from the study. Hemogram was done on automated cell counter analyzer (Sysmex XP 100) which included hemoglobin, hematocrit, total leucocyte count (TLC), differential leucocyte count (DLC) and platelets count. Platelets counts were cross checked on stained smears. Hematocrit raised $>20 \%$ of normal was considered as hemoconcentration. Leukopenia was taken as total leucocyte count $>20 \%$ of normal was considered as hemoconcentration. Leukopenia was taken as total leucocyte count $<4,000 / \mathrm{mm} 3$. Thrombocytopenia was taken as platelets count $<1,00,000 / \mathrm{mm} 3$. Biochemical parameters included serum Aspartate aminotransferase (AST), Alanine aminotransferase (ALT), were done on Cobas c 311 from Roche (Hitachi) biochemistry machine.

Statistical analysis

The recorded data was compiled and entered in a spreadsheet computer program (Microsoft excel 2007) and then exported to data editor page of SPSS version 15 (SPSS Inc., Chicago, Illinois, USA). For all tests, confidence level and level of significance were set at $95 \%$ and $5 \%$ respectively.

\section{Results}

Most of the cases (47.5\%) were seen in the 20-30 year age group followed by 40-50 years $20.83 \%$, 30-40 years $15 \%$. Majority of the patients were males $78(65 \%)$ compared to females, $42(35 \%)$ and the male to female ratio was $1.8: 1$. (Table 1). Fever was the most common presentation and was seen in 58 cases $(48.33 \%)$ cases and followed by Fever and Myalgia 14.17\%, Myalgia $12.5 \%$, Petechiae $9.17 \%$, Fever and Skin rashes, Nausea and vomiting and Fever and Itching. (Table 2) Present study showed hemoglobin range of $6.5 \mathrm{gm} \%$ to $16.5 \mathrm{gm} \% .54(45 \%)$ cases showed $\mathrm{Hb}$ of 9$12 \mathrm{gm} \%$, followed by $38(31.67 \%)$ cases showed $\mathrm{Hb}$ of $12-$ $15 \mathrm{gm} \%, 9(7.5 \%)$ had $\mathrm{Hb}$ of below $9 \mathrm{gm} \%$ and $19(15.83$ $\%$ ) had $\mathrm{Hb}$ of above $15 \mathrm{gm} \%$ (Table - 3). In the present study, 63(52.5\%) cases showed hematocrit of $25-35 \%$ and $35(29.17 \%)$ showed hematocrit of $35-45 \%$. Raised hematocrit $(>45 \%)$ was noted in $22(18.33 \%)$ of patients at presentation. The total leukocyte count ranged from 1500 cells/cumm to $>11000$ cells/cumm. Leucopenia with less than 4000 cells/cumm was present in $21(17.5 \%)$ cases, count of 4000- 11000 cells/cu mm seen in 81(67.5\%) cases and $>11000$ / cumm was seen in 18 cases $(15 \%)$ as per Table 4.In the present study out of 120 cases of dengue fever, $87.5 \%$ cases had thrombocytopenia and $12.5 \%$ cases had severe thrombocytopenia $(<20,000 /$ cumm) with bleeding manifestations. Serum AST and ALT were elevated in 83 $(69.17 \%)$ cases and were normal in $37(30.83 \%)$ cases. In the present study, hepatomegaly was noted in $42(35 \%)$ and splenomegaly was seen in $18(15 \%)$ of cases.

Table 1: Demographic Profile of Patients

\begin{tabular}{|c|c|c|}
\hline Gender & No. of patients =120 & \% \\
\hline Male & 78 & 65 \\
\hline Female & 42 & 35 \\
\hline Age (Years) & & \\
\hline Below 20 & 9 & 7.5 \\
\hline $20-30$ & 57 & 47.5 \\
\hline $30-40$ & 18 & 15 \\
\hline $40-50$ & 25 & 20.83 \\
\hline $50-60$ & 8 & 6.67 \\
\hline Above 60 & 3 & 2.5 \\
\hline Total & 120 & $100 \%$ \\
\hline
\end{tabular}

Table 2: Distribution of Clinical features

\begin{tabular}{|c|c|c|}
\hline Clinical features & No. of cases & \% \\
\hline Fever & 58 & 48.33 \\
\hline Myalgia & 15 & 12.5 \\
\hline Fever and Myalgia & 17 & 14.17 \\
\hline Headache & 3 & 2.5 \\
\hline Nausea and vomiting & 5 & 4.17 \\
\hline Fever and Skin rashes & 9 & 7.5 \\
\hline Petechiae & 11 & 9.17 \\
\hline Fever and Itching & 2 & 1.67 \\
\hline Total & 120 & $100 \%$ \\
\hline
\end{tabular}

Table 3: Distribution of study population by hemoglobin and hematocrit level

\begin{tabular}{|c|c|c|c|c|c|}
\hline \multicolumn{3}{|c|}{ Hemoglobin level } & \multicolumn{3}{c|}{ Hematocrit } \\
\hline Hb (gm/dl) & No. of cases & \% & Hct (\%) & No. of cases & \% \\
\hline Below 9 & 9 & 7.5 & Below 25 & - & - \\
\hline $9-12$ & 54 & 45 & $25-35$ & 63 & 52.5 \\
\hline $12-15$ & 38 & 31.67 & $35-45$ & 35 & 29.17 \\
\hline Above 15 & 19 & 15.83 & $45-55$ & 22 & 18.33 \\
\hline Total & 120 & $100 \%$ & Total & 120 & $100 \%$ \\
\hline
\end{tabular}


Table 4: Distribution of cases according to total leukocyte count and platelet count

\begin{tabular}{|c|c|c|c|c|c|}
\hline \multicolumn{2}{|c|}{ Total leukocyte count } & \multicolumn{3}{c|}{ Platelet count } \\
\hline TLC (cells/cumm) & No. of cases & $\%$ & Platelet (cells/cumm) & No. of cases & \% \\
\hline$<1500$ & - & - & $<20,000$ & 15 & 12.5 \\
\hline $1500-4,000$ & 21 & 17.5 & $20,000-50,000$ & 68 & 56.67 \\
\hline $4000-11,000$ & 81 & 67.5 & $50,000-1.4$ lakh & 19 & 15.83 \\
\hline$>11,000$ & 18 & 15 & $>1.5$ lakhs & 18 & 15 \\
\hline Total & 120 & $100 \%$ & Total & 120 & $100 \%$ \\
\hline
\end{tabular}

In our study most of the cases $(47.5 \%)$ were seen in the 20 30 year age group followed by $40-50$ years $20.83 \%, 30-40$ years $15 \%$. Deshwal, et al. ${ }^{[12]}$ studied a total of 515 patients of Dengue. In their study too maximum patients were in 21 40 year age group $(62.91 \%)$. Vibha, et al. ${ }^{[13]}$ studied 100 patients, and observed $49(49 \%)$ to be in the 15 to 25 year age group followed by $33(33 \%)$ cases in the 26 to 35 years age group. Meena, et al. ${ }^{[14]}$ (12 did a randomized study of 100 patients with Dengue fever. According to age, maximum cases (29\%) were in 21-30 years and rest (27\%) were in $15-20$ years, $(21 \%)$ were in $31-40$ years, $(16 \%)$ were in 41-50 years and (7\%) in 51- 60 years. Ahmed, et al. ${ }^{[15]}$ $(n=205)$ observed the age range for dengue as 10-65 years and the mean age was 31.29 years $(\mathrm{SD} \pm 13.65)$. Our findings compare well with the observations of the above authors.

Majority of the patients were males 78 (65\%) compared to females, $42(35 \%)$ and the male to female ratio was 1.8:1. Deshwal, et al. ${ }^{[12]}$ and Vibha, et al. ${ }^{[13]}$ too observed a male predominance in their studies with $72.8 \%$ and $70 \%$ male patients respectively. The male to female ratio was 1.7:1 in Vibha, et al. ${ }^{[13]}$ study. In the study by Ahmed, et al. ${ }^{[15]}$ the number of males was $193(94.15 \%)$, while females were 12 $(5.85 \%)$ with male to female ratio of $9: 1$ approximately. Meena, et al. [14] $(\mathrm{n}=100)$ also observed a male predominance with 63 cases $(63 \%)$ and $37(37 \%)$ female patients. Our findings correlate well with the above authors. The male predominance can be explained by the fact that usually it's the male population that has excess outdoor activity and the likelihood of being exposed to the vector mosquito bites.

Fever was the most common presentation and was seen in 58 cases $(48.33 \%)$ cases and followed by Fever and Myalgia $14.17 \%$, Myalgia $12.5 \%$, Petechiae $9.17 \%$, Fever and Skin rashes, Nausea and vomiting and Fever and Itching. In the study by Deshwal, et al. ${ }^{[12]}$ fever was universal followed by headache $(94.75 \%)$, myalgia $(90.67 \%)$, conjunctival injection $(39.41 \%)$, morbilliform skin rash $(37.86 \%)$, abdominal pain $(24.46 \%)$, retro-orbital pain $(18.25 \%)$, itching predominantly localized to palmar and plantar aspects of hands and feet (13.39\%). In the study by Vibha, et al. ${ }^{[13]} 95(95 \%)$ of the patients had fever as presenting symptom. Other symptoms were myalgia in $70(70 \%)$ cases, arthralgia in $60(60 \%)$ cases and headache in $50(50 \%)$ cases.

Present study showed hemoglobin range of $6.5 \mathrm{gm} \%$ to $16.5 \mathrm{gm} \%$. $54(45 \%)$ cases showed $\mathrm{Hb}$ of $9-12 \mathrm{gm} \%$, followed by $38(31.67 \%)$ cases showed $\mathrm{Hb}$ of $12-15 \mathrm{gm} \%$, $9(7.5 \%)$ had $\mathrm{Hb}$ of below $9 \mathrm{gm} \%$ and $19(15.83 \%)$ had $\mathrm{Hb}$ of above $15 \mathrm{gm} \%$. In the study by Meena, et al. [14] hemoglobin ranged from 7.5-17.5 g/dl, mean hemoglobin value was $12.6 \mathrm{~g} / \mathrm{dl}$. Hemoglobin level more than $15 \mathrm{gm} \%$ was seen in $6 \%$ cases. Dongre, et al. ${ }^{[16]}$ observed hemoglobin level from $3.6 \mathrm{gm} / \mathrm{dl}$ to $16.7 \mathrm{gm} / \mathrm{dl}$ with a mean of $11.9 \mathrm{gm} / \mathrm{dl}$.
In the present study, 63(52.5\%) cases showed hematocrit of $25-35 \%$ and $35(29.17 \%)$ showed hematocrit of $35-45 \%$. Raised hematocrit $(>45 \%)$ was noted in $22(18.33 \%)$ of patients at presentation. Deshwal, et al. ${ }^{[12]}$ observed raised hematocrit of $>47 \%$ in $20.7 \%$ of patients at presentation. Vibha, et al. ${ }^{[13]}$ observed $>40 \%$ hematocrit in $28(28 \%)$ cases. In present study, hematocrit ranged from $20 \%$ to $51 \%$. The mean hematocrit value of dengue positive cases in our study was $39.08 \%$. In DHF and DSS, an increase in hematocrit levels was noted and was above $45 \%$. Dongre, et al. ${ }^{[16]}$ observed an increased hematocrit of $>40 \%$ in only 16 patients.

The total leukocyte count ranged from 1500 cells/cumm to $>11000$ cells/cumm. Leucopenia with less than 4000 cells/cumm was present in $21(17.5 \%)$ cases, count of 4000- 11000 cells/cu $\mathrm{mm}$ seen in $81(67.5 \%$ ) cases and $>11000$ / cumm was seen in 18 cases (15\%). In Deshwal, et al. ${ }^{[12]}$ study leucopenia was noticed in around $20.19 \%$ of cases. In Meena, et al. ${ }^{[14]}$ study total leukocyte count ranged from 1310 to $16700 \mathrm{cell} / \mathrm{mm}^{3}$, with mean total leukocyte count of 4701 cells/cumm. A total leukocyte count of less than 4,000 cell/cumm was present in $51(51 \%)$ patients whereas, a total leukocyte count of more than 11,000 cell/cumm was present in $4(4 \%)$ patients. Almost $45 \%$ patients had total leukocyte counts between the normal range. Dongre, et al. [16] observed leucopenia (total leucocyte counts $<4000 / \mathrm{cumm}$ ) in 81 cases and normal count (count between 4000 to11000/cumm) in 111 cases. Leucopenia with lymphocytosis was seen in 40 patients.

In the present study out of 120 cases of dengue fever, $87.5 \%$ cases had thrombocytopenia and $12.5 \%$ cases had severe thrombocytopenia $\quad(<20,000 / \mathrm{cumm}) \quad$ with bleeding manifestations.

Deshwal, et al. ${ }^{[12]}$ observed a platelet count of 50,000/cumm at presentation in $69.5 \%$ of cases, though it kept on falling further during hospitalization under observation. In their study minimum platelet count noted was $8,000 /$ cumm. In Meena, et al. ${ }^{[14]}$ study, $(\mathrm{n}=100), 90(90 \%)$ cases had thrombocytopenia, in which 61 patients had platelet count between 20,000-60,000. Out of these 61 patients, seven patients $(11.47 \%)$ had bleeding manifestation. Dongre, et al. ${ }^{[16]}$ observed thrombocytopenia, platelet count $<10,0000$ in 112 patients. Six cases had counts less than 20000/cumm, 32 cases had counts between 20,000- 50,000/cumm, 42 cases had counts between 50,000-75000/cumm and 129 cases had counts more than 75000/cumm. Serum AST and ALT were elevated in $83(69.17 \%)$ cases and were normal in $37(30.83 \%)$ cases. Deshwal, et al. ${ }^{[12]}$ noted raised liver serum transaminases in $88.54 \%$ of their patients.

In the present study, hepatomegaly was noted in $42(35 \%)$ and splenomegaly was seen in 18

(15\%) of cases. Deshwal, et al. ${ }^{[12]}$ too reported hepatomegaly in $14.75 \%$ and splenomegaly in $13.20 \%$ of their cases. 


\section{Conclusion}

Hemoconcentration, leukopenia, thrombocytopenia, and raised liver enzymes SGOT and SGPT along with reactive/ plasmacytoid lymphocytes on peripheral smear gives enough clues to test for dengue serology so that dengue cases can be diagnosed in their initial stages.

\section{References}

1. World Health Organisation. Dengue: Guidelines for Diagnosis, Treatment, Prevention and Control, New Edition, World Health Organization and TDR for research on diseases of poverty. 2009.

2. Azin FRFG, Goncalves RP, Pitombeira MHS, Lima DM, Branco IC. Dengue: profile of hematological and biochemical dynamics. Rev Bras Hematol Hemoter 2012;34(1):36-41.

3. Siqueira JB, Martelli SMT, Coelho GE, Simplício ACR, Hatch DL. Dengue and dengue hemorrhagic fever, Brazil, 1981 2002. Emerging infectious diseases 2005;11(1):48-53

4. Oishi K, Saito M, Mapua CA, Natividad FF. Dengue illness: clinical features and pathogenesis. J Infect Chemother 2007;13(3):125-33.

5. Banerjee M, Chatterjee T, Choudhary GS, Srinivas V, Kataria VK. Dengue: A clinico-hematological profile. Med J Armed Forces India 2008;64:333-6.

6. Nathan MB, Dreger RD, Guzman M. Epidemiology, burden of disease and transmission. In: Dengue Guidelines for Diagnosis, Treatment, Prevention and Control. New ed. Geneva, PA: WHO Press 2009.

7. Potts JA, Rothman AL. Clinical and laboratory features that distinguish dengue from other febrile illnesses in endemic populations. Trop Med Int Health 2008;13:1328-40.

8. Ageep AK, Malik AA, Elkarsani MS. Clinical presentations and laboratory findings in suspected cases of dengue virus. Saudi Med J 2006;27(11):1711-3.

9. Butt N, Abbassi A, Munir SM, Ahmad SM, Sheikh QH. Haematological and biochemical indicators for early diagnosis of dengue viral infections. J Coll Phys Surg Pak 2008;18:282-5.

10. Chen RF, Yang KD, Wang L, Liu JW, Chiu CC, Cheng JT. Different clinical and laboratory manifestations between dengue haemorrhagic fever and dengue fever with bleeding tendency. Trans R Soc Trop Med Hyg. 2007;101(11):1106-13.

11. Lin CF, Wan SW, Cheng H J, Lei HY, Lin YS. Autoimmune pathogenesis in dengue virus infection. Viral Immunol 2006;19(2):127-32.

12. Deshwal R, Qureshi MI, Singh R. Clinical and Laboratory Profile of Dengue Fever. Journal of The Association of Physicians of India 2015,63.

13. Gajera VV, Sahu S, Dhar R. Study of Haematological Profile of Dengue Fever and its Clinical Implication. Annals of Applied Bio-Sciences 2016;3(3):2455-96.

14. Meena KC, Jelia S, Meena S, Arif M, Ajmera D, Jatav VS. A study of hematological profile in dengue fever at a tertiary care center, Kota Rajasthan. Int J Adv Med 2016;3(3):621-624.

15. Ahmed F, Hussain Z, Ali Z. Clinical and hematological profile of patients with dengue fever. J. Med. Sci Peshawar, Print 2014;22(1):17-20.

16. Dongre T, Karmarkar P. Hematological Parameters and Its Utility in Dengue-A Prospestive Study. IOSR
Journal of Dental and Medical Sciences 2015;14(2):3134. 